\title{
Suppressive Effect of Cocoa Powder on Atherosclerosis in Kurosawa and Kusanagi-hypercholesterolemic Rabbits
}

\author{
Tohru Kurosawa', Fumi Itoh'1, Aiko Nozaki', Yoshihisa Nakano, Shin-ichiro Katsuda ${ }^{3}$, \\ Naomi Osakabe ${ }^{2}$, Hirokazu Tsubone ${ }^{4}, K_{\text {Kazuo Kondo }}^{5}$, and Hiroshige Itakura ${ }^{6}$ \\ ${ }^{1}$ Toxicology Laboratory, Pharmaceutical Development Department, Meiji Seika Kaisha, Ltd., Kanagawa, Japan. \\ ${ }^{2}$ Nutritional Science Center, Health and Bioscience Laboratories, Meiji Seika Kaisha, Ltd., Saitama, Japan. \\ ${ }^{3}$ Department of Physiology, Fukushima Medical University School of Medicine, Fukushima, Japan. \\ ${ }^{4}$ Department of Comparative Pathophysiology, Graduate School of Agricultural and Life Sciences, The University \\ of Tokyo, Tokyo, Japan. \\ ${ }^{5}$ Institute of Environmental Science for Human Life, Ochanomizu University, Tokyo, Japan. \\ ${ }^{6}$ Department of Food Science, Ibaraki Christian University, Ibaraki, Japan.
}

\begin{abstract}
We investigated the suppressive effect of cocoa powder (cacao polyphenol content: $\mathbf{7 . 8 \% )}$ on atherosclerosis in a spontaneous familial hypercholesterolemic model, Kurosawa and Kusanagi-hypercholesterolemic (KHC) rabbits. Six-month dietary administration of cocoa powder had no effects on body weight, hematology or blood chemistry parameters or a lipid profile in KHC rabbits. Antioxidative activity of lowdensity lipoprotein (LDL) was observed in the 2nd month and 3rd month of administration. Thiobarbituric acid reactive substances (TBARS), the marker of lipid peroxidation, in plasma were decreased in the cocoa powder treated group from the 2nd month of administration during the study period compared to that in the control group. The area of atherosclerotic lesions in th aorta was significantly smaller in the cocoa powder group $(30.87 \%)$ than in the control $(52.39 \%)$. Tissue cholesterol content also tended to decrease. Distensibility of the aortic wall was improved significantly in the cocoa powder treated group due to decreases in fatty streaks and intimal thickening compared to that in the control group. These results suggest that cocoa powder has suppressive effect on development of atherosclerotic lesions. We consider that antioxidative activity of polyphenols rich in cocoa powder may be a key factor for the anti-atherosclerotic effect. J Atheroscler Thromb, 2005; 12: 20-28.
\end{abstract}

Key words: Polyphenols, KHC rabbit, LDL oxidation, Antioxidant

\section{Introduction}

Epidemiological studies revealed that the intake of food abundant in polyphenols, antioxidative substances, reduces mortality risks from cardiovascular diseases (13). Cacao beans (Theobroma cacao) are the main ingre-

Address for correspondence: Tohru Kurosawa, Toxicology Laboratory, Pharmaceutical Development Department, Meiji Seika Kaisha, Ltd., 760 Morooka-cho, Kohoku-ku, Yokohama 2228567, Japan.

E-mail: toru_kurosawa@meiji.co.jp

Received June 23, 2004.

Accepted for publication August 16, 2004 dient for chocolates and cocoa drinks. Cacao beans are rich in polyphenols including catechins and their olygomers such as procyanidins (4-6). Crude cacao polyphenol fractions have been reported so far to possess in vitro antioxidative activity $(7,8)$ and suppressive

\footnotetext{
Abbreviations:

KHC rabbit: Kurosawa and Kusanagi-hypercholesterolemic rabbit

LDL: low-density lipoprotein

TBARS: thiobarbituric acid reactive substances

$\mathrm{FH}$ : familial hypercholesterolemia

V-70: 2,2'-azobis(4-methoxy)-2,4-dimethylvaleronitrile

$\mathrm{EM}_{0.5}$ : elastic modulus of the wall at a strain of 0.50
} 
activity of LDL oxidation in cholesterol-fed rabbits (9). A clinical study in healthy volunteers disclosed that daily intake of cocoa powder decreased susceptibility of LDL to oxidation (10). LDL oxidation is considered to play an important role in development of atherosclerosis (11), and dietary intake of cacao polyphenol is thereby expected to suppress it. In the present study, we examined antiatherosclerotic effect of cocoa powder in KHC rabbits, the animal model of familial hypercholesterolemia (FH) that exhibits hypercholesterolemia from birth and spontaneously develops atherosclerosis (12).

\section{Materials and Methods}

\section{Cocoa powder}

Fermented and dried cacao beans were imported from Ecuador. The beans were processed in Meiji Seika Kaisha, Ltd. (Tokyo, Japan) through roasting, grinding and compressing to prepare cocoa powder. The composition of this cocoa powder is shown in Table 1.

\section{Animals and diets}

Six male and 6 female homozygous $\mathrm{KHC}$ rabbits (body weight range: $1.8-2.3 \mathrm{~kg}$ ) were purchased from Japan Laboratory Animals Inc. (Tokyo, Japan) at the age of 3 months. The animals were individually housed under controlled environment (room temperature: $21-25^{\circ} \mathrm{C}$, relative humidity: $45-65 \%, 12$-hour dark and light cycle, more than 10 times ventilation per hour). The control animals received commercially available standard diets (RC-4, Oriental Yeast, Tokyo, Japan). The animals in the treatment group received the admixture of the standard diets and cocoa powder by $10 \%$. The mixed diets were stored at $-20^{\circ} \mathrm{C}$ until immediately before feeding.

Table 1. General composition per $10 \mathrm{~g}$ of the cocoa powder.

\begin{tabular}{lc}
\hline Component & Content \\
\hline Carbohydrate & $1.6 \mathrm{~g}$ \\
Fat & $1.47 \mathrm{~g}$ \\
Protein & $2.11 \mathrm{~g}$ \\
Fiber & $2.58 \mathrm{~g}$ \\
Mineral & $0.81 \mathrm{~g}$ \\
Caffeine & $34.3 \mathrm{mg}$ \\
Theobromine & $0.25 \mathrm{~g}$ \\
$\alpha$-Tocopherol & $18.9 \mu \mathrm{g}$ \\
$\gamma$-Tocopherol & $0.3 \mathrm{mg}$ \\
Total polyphenol & $0.78 \mathrm{~g}$ \\
\hline
\end{tabular}

\section{Study design}

The rabbits were divided into 2 groups so as to have no group differences in body weight, plasma total cholesterol, or triglyceride (3 males and 3 females for each of the control and treatment groups). The control group received $100 \mathrm{~g}$ of the diets per day and the cocoa powder group received $110 \mathrm{~g}$, and both groups were provided with drinking tap water ad libitum. The animals were clinically observed and food consumption was measured every day. The measurement of body weights, hematology, blood chemistry and LDL oxidation were performed 1 month after the initiation of the study and onward at 1 month intervals. The animals were euthanised by overdose of pentobarbital at the end of the 6-month administration study of cocoa powder.

\section{Hematology and blood chemistry}

Hematology [red blood cell count (RBC), hemoglobin $(\mathrm{Hb})$, hematocrit, mean corpuscular volume, mean corpuscular hemoglobin, mean corpuscular hemoglobin concentration, platelet count (PIt), white blood cell count (WBC)] was performed with a cell counter (ADVIA 120, Bayer, USA) using the blood collected from the auricular vein under EDTA-3K treatment. Blood chemistry [total protein (TP), albumin, glucose (Glu), blood urea nitrogen (BUN), creatinine (Cre), aspartate aminotransferase (AST), alanine aminotransferase (ALT), lactate dehydrogenase $(\mathrm{LDH})$, creatine phosphokinase (CPK), alkaline phosphatase (ALP), calcium] was performed with an autoanalyzer (COBAS FARA II, Roche, Switzerland) and commercially available kits (Wako Pure Chemical, Osaka, Japan) using the plasma centrifuged from the heparintreated blood sample. Sodium, chlorine and potassium were measured with the ionic membrane electrode method using EA03 (A\&T, Tokyo, Japan).

\section{Measurement of plasma lipid levels}

The plasma centrifuged from the EDTA-3K-treated blood sample was examined for total cholesterol, triglyceride, phospholipid and TBARS. The total cholesterol, triglyceride and phospholipid were measured enzymatically using COBAS FARA II (Roche) and commercially available kits (Wako Pure Chemical). TBARS was measured in accordance with the method of Yagi (13) using a fluorophotometer (Model 650-10S; Hitachi, Tokyo, Japan) and a commercially available kit (Wako Pure Chemical).

\section{Evaluation of antioxidation in LDL}

LDL oxidation was measured with the method developed by Esterbauer et al. (14) and Hirano et al. (15). The plasma was obtained from the EDTA-3K treated blood sample (final concentration: $1 \mathrm{mg} / \mathrm{ml}$ ) and ultracentrifuged to obtain LDL $(d=1.019-1.063 \mathrm{~g} / \mathrm{ml})$. LDL fraction was confirmed to have a single peak in agarose gel electro- 
phoresis. To the LDL fraction (final concentration of protein: $70 \mu \mathrm{g} / \mathrm{ml})$, an oxidant $\mathrm{V}-70$ (2,2'-azobis(4-methoxy)2,4-dimethylvaleronitrile) was added to be $200 \mu \mathrm{M}$ and incubated at $37^{\circ} \mathrm{C}$. The formation of conjugated diene was monitored at $234 \mathrm{~nm}$ using Beckman Model DU640 spectrophotometer to obtain the lag time, that is the time until the conjugated diene started increasing.

\section{Determination of lesioned area}

The aorta was excised from the origin to the iliac bifurcation. After fats and tissues adhering to the adventitia were removed, the aorta was cut open longitudinally and the luminal surface was Xerox-copied. Atherosclerotic lesions were traced carefully and the area ratio of atherosclerotic lesions to the total area was calculated by image analysis (NIH Image 1.62; National Institutes of Health, USA).

\section{Measurement of lipid contents in the aorta}

Each aorta was divided into three segments, that is aortic arch (from the origin to the 1st intercostal arteries), thoracic aorta (between the 1st to 8th intercostal arteries), and abdominal aorta (from the 8th intercostal arteries to the iliac bifurcation). Each segment of the aorta was homogenized with $1.14 \% \mathrm{KCl}$ containing $50 \mathrm{mM}$ deferoxamine. Cholesterol was extracted in a mixed solution of methanol and chloroform to determine total cholesterol using a commercially available kit (Wako Pure Chemical). To the homogenate, a mixed solution of $8.1 \%$ sodium dodecyl sulfate and $20 \%$ acetic acid, and $0.8 \%$ thiobarbituric acid were added. It was then boiled and TBARS were extracted in a mixed solution of $n$-butanol and pyridine to determine intensity of fluorescence.

\section{Static rheological properties of the aorta}

Circumferential wall strips of $3.0 \mathrm{~mm}$ in width were excised from the ascending aorta and descending portion of aortic arch (about $2 \mathrm{~cm}$ peripheral to the left subclavian bifurcation) where atherosclerotic lesions were relatively marked. Both ends of each strip were mounted into chucks fixed to a tensile testing instrument (TOM-30J, Minebea Co., Ltd., Tokyo, Japan), immersed into the Locke's solution at $37^{\circ} \mathrm{C}$, and stretched at a speed of $4.17 \mathrm{~mm} / \mathrm{sec}$ until tension reached $20-30 \mathrm{~g}$ as shown in the previous report (16). Immediately after the stressstrain characteristics test, the strip was relaxed to the initial length, detached from the chucks and weighed precisely. Stress value $(\tau)$ was calculated from the following formula assuming Poisson's ratio of the vascular wall as $0.5: \tau=1.06 \times \mathrm{T} / \mathrm{W} \times \mathrm{L}_{0}\left(1+\Delta \mathrm{L} / \mathrm{L}_{0}\right)$, where 1.06, $\mathrm{T}, \mathrm{W}, \mathrm{L}_{0}$ and $\Delta \mathrm{L}$ were density of the wall, wall tension (g), sample weight $(\mathrm{g})$, initial length of the strip $(\mathrm{cm})$ and increment from the initial length $(\mathrm{cm})$, respectively. Wall thickness (h) at the initial length was calculated from the following formula: $h=(W / 1.06) /\left(L_{0} \times W d\right)$, where Wd was the width of strip (cm). Incremental elastic modulus of the wall at a strain of $0.50\left(\mathrm{EM}_{0.5}\right)$ with respect to the unstressed length was determined as the mean gradient of the stress-strain curve between strains of 0.55 and 0.45 , and calculated as follows: $\mathrm{EM}_{0.5}=\left(\sigma_{0.55}-\sigma_{0.45}\right) /\left(\varepsilon_{0.55}-\varepsilon_{0.45}\right)$, where $\sigma_{0.55}$ and $\sigma_{0.45}$ were stress at strains of 0.55 and 0.45 and $\varepsilon_{0.55}$ and $\varepsilon_{0.45}$ strains of 0.55 and 0.45 , respectively (16). Each strip of the aorta used in the tensile test was fixed in $10 \%$ neutral buffered formalin solution. It was then embedded into paraffin, sectioned at $3 \mu \mathrm{m}$ in thickness and stained with hematoxylin-eosin or elastica van Gieson.

\section{Statistical analysis}

All data are expressed in mean \pm S.E. For determination of a significant difference from the control group, Student's $t$-test was used. For determination of a significant difference from the value prior to administration, paired $t$-test was performed. $p<0.05$ was regarded as significant.

\section{Results}

There was no difference in all data mentioned below between males and females in each group.

\section{General conditions}

Throughout the study period, there were neither adverse effects of cocoa powder in clinical observation nor decrease in food consumption. There was no significant difference in body weight between the control and cocoa powder treated groups. The body weights were 2.1 $\pm 0.1 \mathrm{~kg}$ and $2.0 \pm 0.1 \mathrm{~kg}$ before the start of study, and $3.3 \pm 0.1 \mathrm{~kg}$ and $3.1 \pm 0.1 \mathrm{~kg}$ after 6 months administration, respectively. In any test item for hematology or blood chemistry, no significant difference was noted between the control and cocoa powder groups (Table 2).

\section{Plasma lipid levels}

The level of plasma total cholesterol in the cocoa powder group was equivalent to that in the control. There was no significant difference in triglyceride or phospholipid between the control and cocoa powder groups. The level of plasma TBARS in the cocoa powder group was significantly decreased compared to the control from the 2nd month of administration (Fig. 1).

\section{Antioxidative effect in LDL}

The lag time was extended in the cocoa powder group in the 2nd month and 3rd month of administration compared to the value prior to the start of administration. When compared to the control $(54.4 \pm 2.2 \mathrm{~min})$, the lag time in the cocoa powder group was significantly prolonged to $72.0 \pm 3.1 \mathrm{~min}$ in the $3 \mathrm{rd}$ month of administration (Table 3). 


\section{Area of atherosclerotic lesions in the aorta}

Photocopies of the aortic intima surfaces are shown in Fig. 2. In each location, the percent area of lesions to the total intimal area tended to decrease in the cocoa powder group. The ratios in the whole aorta and aortic arch in this group were significantly lower than those in the control (Table 4).

\section{Lipid contents in the aorta}

In the cocoa powder group, the total cholesterol level in the aorta tended to be lower in any location compared to the control, however the difference was not significant. The level of TBARS also tended to be low in any

Table 2. Blood chemical and hematological findings in $\mathrm{KHC}$ rabbits fed with cocoa powder.

\begin{tabular}{|c|c|c|c|c|}
\hline \multirow{2}{*}{ Parameter } & \multicolumn{2}{|c|}{ Control } & \multicolumn{2}{|c|}{ Cocoa powder } \\
\hline & Baseline & 6 months & Baseline & 6 months \\
\hline $\begin{array}{l}\text { RBC } \\
\left(\times 10^{6} / \mu \mathrm{l}\right)\end{array}$ & $6.68 \pm 0.21$ & $6.62 \pm 0.20$ & $6.48 \pm 0.15$ & $6.30 \pm 0.43$ \\
\hline $\mathrm{Hb}(\mathrm{g} / \mathrm{dl})$ & $12.9 \pm 0.3$ & $13.1 \pm 0.4$ & $12.7 \pm 0.1$ & $12.4 \pm 0.2$ \\
\hline $\begin{array}{l}\text { Plt } \\
\left(\times 10^{3} / \mu \mathrm{l}\right)\end{array}$ & $421 \pm 16$ & $402 \pm 4$ & $478 \pm 51$ & $529 \pm 69$ \\
\hline $\begin{array}{l}\text { WBC } \\
\left(\times 10^{3} / \mu \mathrm{l}\right)\end{array}$ & $7.76 \pm 0.37$ & $6.43 \pm 0.68$ & $7.92 \pm 0.39$ & $5.61 \pm 0.93$ \\
\hline TP (g/dl) & $5.9 \pm 0.1$ & $6.9 \pm 0.2$ & $5.9 \pm 0.0$ & $6.5 \pm 0.3$ \\
\hline $\begin{array}{l}\text { Glu } \\
\text { (mg/dl) }\end{array}$ & $129 \pm 3$ & $118 \pm 2$ & $136 \pm 4$ & $121 \pm 3$ \\
\hline $\begin{array}{l}\text { BUN } \\
(\mathrm{mg} / \mathrm{dl})\end{array}$ & $17.8 \pm 0.8$ & $19.2 \pm 1.0$ & $17.3 \pm 1.1$ & $19.9 \pm 0.9$ \\
\hline $\begin{array}{l}\text { Cre } \\
\text { (mg/dl) }\end{array}$ & $0.62 \pm 0.04$ & $0.81 \pm 0.04$ & $0.67 \pm 0.03$ & $0.95 \pm 0.07$ \\
\hline AST (IU/I) & $35 \pm 2$ & $24 \pm 2$ & $45 \pm 6$ & $20 \pm 2$ \\
\hline ALT (IU/I) & $19 \pm 2$ & $21 \pm 2$ & $24 \pm 3$ & $28 \pm 5$ \\
\hline LDH (IU/I) & $88 \pm 11$ & $51 \pm 5$ & $88 \pm 9$ & $44 \pm 2$ \\
\hline CPK (IU/I) & $343 \pm 42$ & $372 \pm 116$ & $390 \pm 30$ & $192 \pm 21$ \\
\hline ALP (IU/I) & $399 \pm 34$ & $49 \pm 4$ & $449 \pm 52$ & $44 \pm 3$ \\
\hline
\end{tabular}

All values are mean \pm S.E.

Numbers of rabbits in the groups are: $n=5-6$ (Control group), $n=6$ (Cocoa powder group).

No significant difference was observed by the Student's $t$ test. location in the cocoa powder group, and a significant decrease was observed in the abdominal aorta (Fig. 3).

\section{Static rheological properties of the aorta}

The tension-strain curve and the stress-strain curve in the circumferential strips excised from the ascending aorta and descending portion of aortic arch are shown in
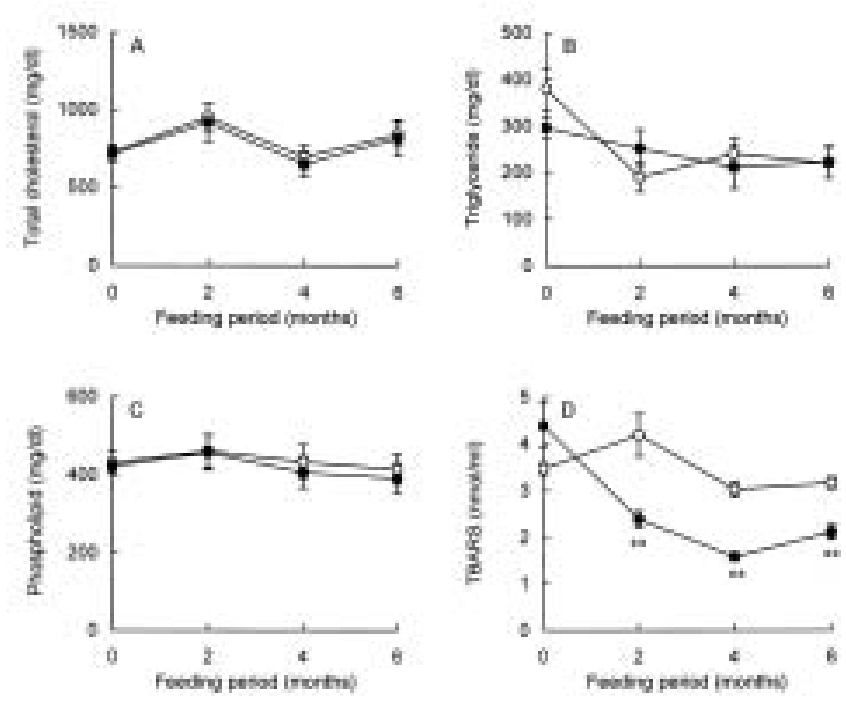

Fig. 1. Plasma lipid levels in $\mathrm{KHC}$ rabbits fed with cocoa powder. Total cholesterol (A), triglyceride (B), phospholipid (C) and TBARS (D) in plasma were determined using commercial kits. Data are expressed as mean \pm S.E. $(n=6)$. Control group $(O)$ Cocoa powder group (๑). Significantly different from control, $* * p<0.01$ (Student's $t$-test).

Table 4. Effect of cocoa powder on the extent of atherosclerotic lesions in aortas of KHC rabbits.

\begin{tabular}{llc}
\hline & \multicolumn{2}{c}{ Area of atherosclerosis (\%) } \\
\cline { 2 - 3 } & \multicolumn{1}{c}{ Control } & Cocoa powder \\
\hline Aortic arch & $76.22 \pm 5.05$ & $55.52 \pm 6.59 *$ \\
Thoracic aorta & $48.76 \pm 12.10$ & $19.53 \pm 5.11$ \\
Abdominal aorta & $37.84 \pm 6.58$ & $25.85 \pm 6.93$ \\
Whole aorta & $52.39 \pm 7.68$ & $30.87 \pm 5.61 *$ \\
\hline
\end{tabular}

All values are mean \pm S.E. $(n=6)$.

Significantly different from control, $* p<0.05$ (Student's $t$ test).

Table 3. Effect of cocoa powder on LDL oxidation in KHC rabbits.

\begin{tabular}{lccccc}
\hline \multirow{2}{*}{ Group } & \multicolumn{5}{c}{ Lag time (minutes) } \\
\cline { 2 - 6 } & Baseline & 2 months & 3 months & 4 months & 6 months \\
\hline Control & $53.5 \pm 1.7$ & $\mathrm{NE}$ & $54.4 \pm 2.2$ & $\mathrm{NE}$ & $36.1 \pm 2.2$ \\
Cocoa powder & $49.8 \pm 2.6$ & $56.7 \pm 2.2^{\S}$ & $72.0 \pm 3.1 * *, \S \S$ & $54.4 \pm 1.9$ & $35.9 \pm 1.3$ \\
\hline
\end{tabular}

All values are mean \pm S.E. $(n=6)$. NE: Not examined. Significantly different from control, $* * p<0.01$ (Student's $t$-test).

Significantly different in prolongation of lag time from baseline, ${ }^{\S} p<0.05, \S \S p<0.01$ (Paired $t$-test). 
Fig. 4 and Fig. 5, respectively. The stress value at each strain in the descending portion of aortic arch was significantly large in the cocoa powder group compared to
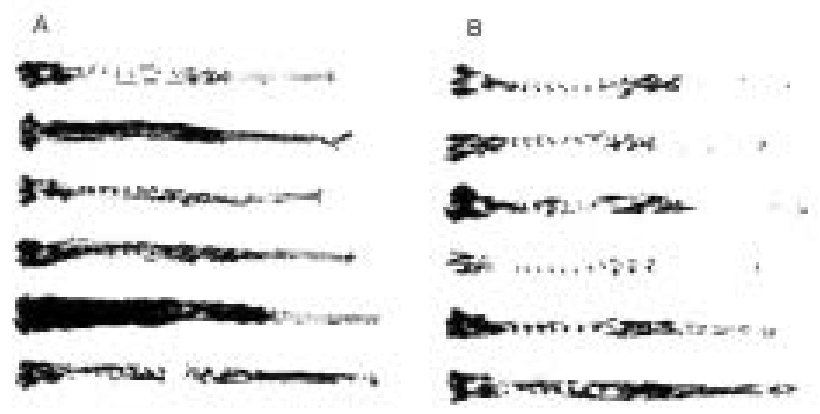

Fig. 2. Xerox-copies of the aortic intima surfaces in KHC rabbits. Atherosclerotic lesions were traced with black. Control group (A); cocoa powder group (B).
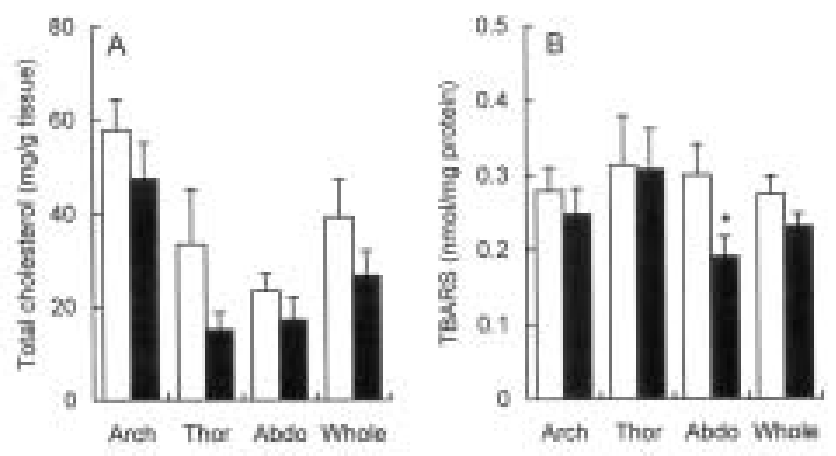

Fig. 3. Effect of cocoa powder on aortic lipid contents in $\mathrm{KHC}$ rabbits. Total cholesterol (A) and TBARS (B) were determined in the aortic arch (Arch), thoracic aorta (Thor), abdominal aorta (Abdo) and whole aorta (Whole). Data are expressed as mean \pm S.E. $(n=6)$. Control group $(\square)$; Cocoa powder group (ם). Significantly different from control, $* p<0.05$ (Student's $t$ test).
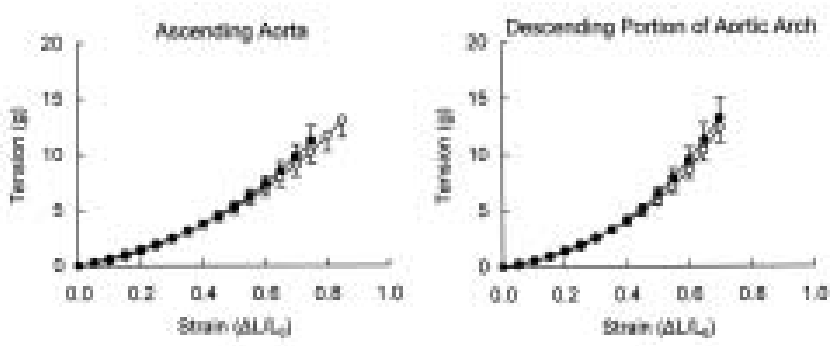

Fig. 4. Tension-strain curves of the circumferential strips of the aortic wall excised from the ascending aorta and descending portion of aortic arch in $\mathrm{KHC}$ rabbits fed with cocoa powder. Data are expressed as mean \pm S.E. $(n=6)$. Control group $(O)$; Cocoa powder group (๑). No significant difference was observed by the Student's $t$-test. $L_{0}$ : Initial length, $\Delta \mathrm{L}$ : Increment from the initial length. that in the control. The wall thickness of the descending portion of aortic arch was significantly small in the cocoa powder group compared to that in the control, and the value of $\mathrm{EM}_{0.5}$ was significantly greater in the cocoa powder group than in the control group (Table 5). In histopathology, thickening of tunica intima in the descending portion of aortic arch was mild in the cocoa powder group compared to that in the control group (Fig. 6).

\section{Discussion}

The administration of cocoa powder by $10 \%$ of the diet prevented development of atherosclerosis in $\mathrm{KHC}$ rabbits. The intake of cocoa powder neither affected plasma cholesterol, triglyceride or phospholipid, nor affected body weight or hematology and blood chemistry parameters. The dosage of cocoa powder in the present study was decided on the basis of a preliminary study (9), in which the short-term $1 \%$ dietary administration of crude polyphenol fraction extracted from cacao beans enhanced resistance against LDL oxidation in cholesterol-

Table 5. Thickness and elastic modulus of the wall at the ascending aorta and descending portion of aortic arch in $\mathrm{KHC}$ rabbits fed with cocoa powder.

\begin{tabular}{|c|c|c|c|}
\hline Parameter & Aortic arch & Control & Cocoa powder \\
\hline \multicolumn{4}{|c|}{ Wall thickness $(\mu \mathrm{m})$} \\
\hline & Ascending & $1,301.4 \pm 62.8$ & $1,231.3 \pm 105.9$ \\
\hline & Descending & $1,240.2 \pm 50.8$ & $739.6 \pm 61.7 * *$ \\
\hline
\end{tabular}

$\mathrm{EM}_{0.5}\left(\times 10^{6} \mathrm{dyn} / \mathrm{cm}^{2}\right)$

$\begin{array}{lll}\text { Ascending } & 0.742 \pm 0.101 & 0.858 \pm 0.060 \\ \text { Descending } & 1.058 \pm 0.130 & 2.165 \pm 0.473^{*}\end{array}$

All values are mean \pm S.E. $(n=6)$. Significantly different from control, $* p<0.05, * * p<0.01$ (Student's $t$-test). $\mathrm{EM}_{0.5}$ : elastic modulus of the wall at a strain of 0.50 .
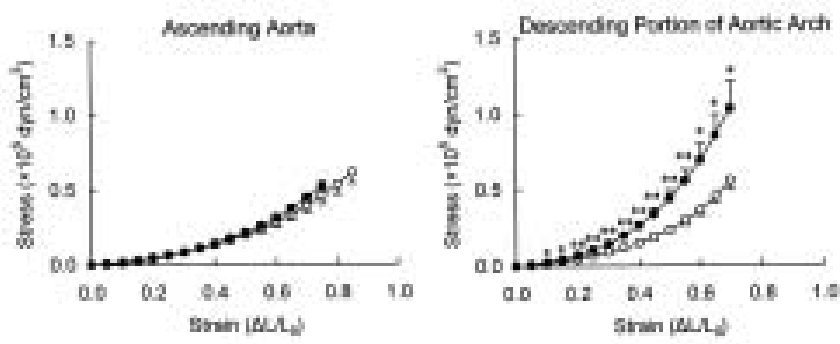

Fig. 5. Stress-strain curves of the circumferential strips of the aortic wall excised from the ascending aorta and descending portion of aortic arch in KHC rabbits fed with cocoa powder. Data are expressed as mean \pm S.E. $(n=6)$. Control group $(O)$; Cocoa powder group ( $(\mathbf{)}$. Significantly different from control, * $p<0.05$, ** $p<0.01$ (Student's $t$-test). $\mathrm{L}_{0}$ : Initial length, $\Delta \mathrm{L}$ : Increment from the initial length. 
fed rabbits. The dosage was then fixed at $10 \%$ admixture of the cocoa powder (total polyphenol content: $7.8 \%$ ), so that the intake of polyphenol would be close to that in the above-mentioned study.

We confirmed that intake of cocoa powder increased resistance against oxidation of LDL, and decreased plasma TBARS in the KHC rabbits. Antioxidative effect of polyphenol that is abundant in cocoa powder was considered to be involved in this mechanism. Polyphenol is abundant in cacao beans. Especially, it is rich in processed foods such as cocoa powder and chocolates. Cacao polyphenols contain monomeric (+)-catechin and (-)-epicatechin, dimeric procyanidin B2, trimeric procyanidin $\mathrm{C} 1$, and tetrameric cinnamtannin $\mathrm{A} 2$. The crude extract fraction that contains these cacao polyphenols was shown to have antioxidative activity $(7,8)$. In vitro studies reported that these compounds trap superoxide and hydroxyradical $(6,17)$, and suppress oxidation stress $(18,19)$. The intake of cocoa powder or crude cacao polyphenol fraction was proved to increase resistance against oxidation of plasma or LDL in rabbits (9), rats $(20,21)$ and humans $(10)$. These polyphenols need to be taken up into bodies to exert their antioxidative activity, however the absorption, metabolism and distribution of them are not well known. Catechin was recently reported to be absorbed efficiently through the digestive tract compared to other flavonoids (22). In healthy volunteers, the intake of cocoa drinks or chocolates led epicatechin and its metabolite to reach their peak in plasma at 1 or 2 hours later $(23,24)$. Baba et al. reported
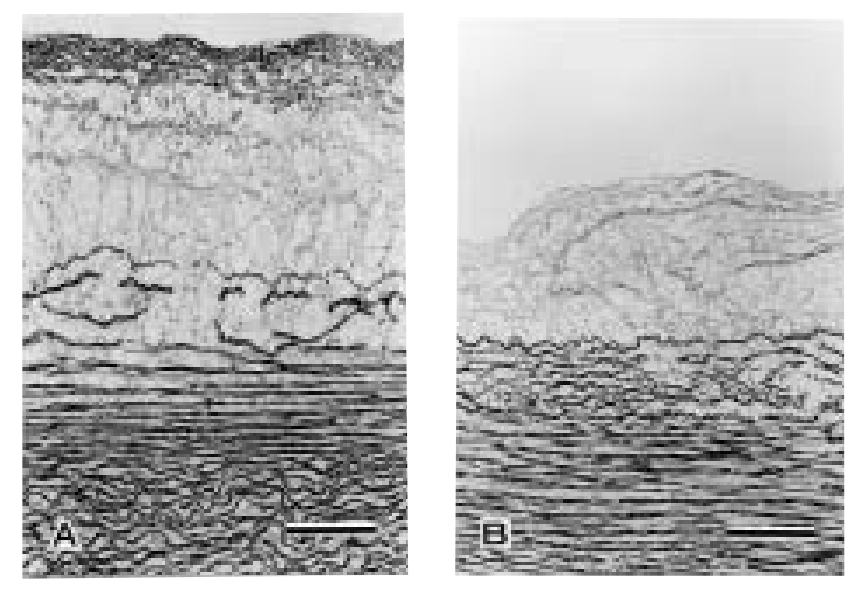

Fig. 6. Microphotographs of the descending portion of aortic arch in KHC rabbits with elastica van Gieson stain. Bar $=100$ $\mu \mathrm{m}$.

A: A control animal. Thickening of the tunica intima mainly due to accumulation of a large amount of foam cells is observed. B: A cocoa powder treated animal. Compared to the control animal, the cocoa powder treated animal shows slight thickening of the tunica intima. the correlation of antioxidative effect and plasma epicatechin concentration in rats treated with cocoa powder (20). In dietary administration of the crude polyphenol fraction to cholesterol-fed rabbits, epicatechin and its metabolite were not detected in plasma, but antioxidative activities were observed (9). It was considered that the low plasma polyphenol concentration was resulting from slow intake of the crude polyphenol fraction by rabbits due to dietary administration. In the present study, polyphenols contained in cocoa powder were administered at almost the same dosage as in the abovementioned study. We did not determine plasma polyphenol concentrations, but a variety of polyphenols presumably existed in rabbit plasma at such concentrations as to exert antioxidative effect. It is not known exactly why enhancement of LDL resistibility against oxidation was transient in cocoa powder-fed $\mathrm{KHC}$ rabbits. The condition of LDL oxidation ex vivo may be too strong to detect antioxidative activity of cacao polyphenols in the present study. Plasma TBARS, which is both the marker of lipid peroxidation and the indirect index of oxidative stress, was, however, decreased in the cocoa powder treated group until the end of administration. We therefore assume that antioxidative effect of polyphenols has been persisted in vivo throughout the study period.

The KHC rabbit is a model animal of familial hypercholesterolemia $(\mathrm{FH})$ that spontaneously develops atherosclerosis, exhibiting hypercholesterolemia from birth due to inheritable defect of LDL receptor, as observed in Watanabe heritable hyperlipidemic (WHHL) rabbits (12). A variety of physiological characteristics in $\mathrm{KHC}$ rabbits have been reported $(12,16)$. In the $\mathrm{KHC}$ rabbits, atherosclerosis in the aorta is extended to the aortic arch and major arterial bifurcations by the age of 3 months, and further descended to the peripheral regions with age (12). In the present study, marked atherosclerotic lesions were observed in the aortas of control animals at the end of the study (at the age of 9 months). In the $\mathrm{KHC}$ rabbits fed cocoa powder, the area of atherosclerotic lesions in the aorta was decreased significantly. Correspondingly, the cholesterol contents in the vascular walls tended to decrease. In the descending portion of aortic arch, the value of wall stress and $\mathrm{EM}_{0.5}$ were significantly greater in the cocoa powder group than in the control group. This means the aortic wall in the control group was more viscoelastic compared to that in the cocoa powder group. Katsuda et al. (16) suggested in the previous report that in the process of remodeling, the aortic wall undergoes "softening" due to accumulation of foam cells in the relatively early stage of atherosclerosis and thereafter increases stiffness with progress of fibrous proliferation or calcification. As foam cells increase, the wall became viscoelastic in the relatively early stage of atherosclerosis. Similar results have been reported in cholesterol-fed cockerels (25) and cholesterol-fed rabbits (26). The co- 
coa powder inhibited accumulation of cholesterol-rich foam cells in the intimal layer, which was considered to be responsible for less viscoelastic properties of the aortic wall.

There is a widely accepted theory that oxidized LDL plays an important role in the onset and development of atherosclerosis $(11,27)$. Some epidemiological studies reported relationship between susceptibility of LDL to oxidation and atherosclerosis in humans $(28,29)$. Suppression of atherosclerosis by antioxidants has been proven in human and animal models. Inverse correlation between intake of antioxidants and cardiovascular diseases were shown in epidemiological studies (1-3). In FH model animals such as WHHL rabbits and KHC rabbits, natural antioxidative substances like vitamin E (30) and taurine (31), herbal medicines containing flavonoid $(32,33)$, and hypocholesterolemic agent possessing antioxidative activity, probucol $(34,35)$ were reported to suppress development of atherosclerosis. These antioxidative substances exert anti-atherosclerotic effect without decreasing plasma cholesterol. Yamakoshi et al. (36) reported that grape seed polyphenols significantly suppressed the susceptibility of LDL to oxidation in cholesterol-fed rabbits, and macrophage-originated foam cells filled with oxidized LDL were decreased in the thickened lesion of tunica intima without affecting plasma cholesterol level. As explained in the above, it is considered that antioxidative substances suppress LDL oxidation, which will not thereby induce foam cell formation. As is the case with these reports, LDL oxidation was suppressed and plasma lipid peroxide was decreased in the cocoa powder treated rabbits. There were no changes in plasma lipid, and atherosclerotic lesion was improved. We therefore considered that polyphenols of cocoa powder suppressed oxidation of LDL and other lipids, which prevented foam cell formation and inhibited the generation and development of atherosclerosis.

Cacao polyphenols have a good profile for healthy blood vessels, for example, inhibition of platelet aggregation (37), vasodilative activity through controlling the levels of eicosanoids and $\mathrm{NO}(38,39)$, and regulation of cytokine production (40) as well as antioxidative effects on LDL. These effects might also contribute to anti-atherosclerotic effect observed in the present study. Cocoa powder contains not only polyphenols but also dietary fibers, a lot of minerals, theobromine possessing a variety of pharmacological effects, and so on (41). Dietary fibers are known to decrease serum lipid (42), however there was no change in lipids in the cocoa powder-fed $\mathrm{KHC}$ rabbits in our study during its 6-month study period. Minerals such as potassium, calcium and magnesium are involved in maintaining vascular functions and lowering blood pressure $(43,44)$. Additionally, magnesium has been reported to suppress atherosclerosis through its lipid-lowering activity and antioxidative effect $(45,46)$.
Recently, Azam et al. (47) reported xanthine derivatives such as theobromine or caffeine have possibility to act as antioxidative substances. We also assume these elements may be partially involved in suppressive effects on development of atherosclerosis in $\mathrm{KHC}$ rabbits. Further studies are thereby required to confirm suppressive effects of cacao polyphenols on atherosclerosis.

In conclusion, cocoa powder suppressed the development of atherosclerosis in the $\mathrm{FH}$ model animals, $\mathrm{KHC}$ rabbits. Our results suggested that the anti-atherosclerotic effect of cocoa powder was involved in the suppression of LDL oxidation. The intake of polyphenol-rich processed food of cacao beans like cocoa powder and chocolates would be beneficial to prevent the onset of atherosclerosis and cardiovascular diseases.

\section{References}

(1) Hertog MG, Feskens EJ, Hollman PC, Katan MB, and Kromhout D: Dietary antioxidant flavonoids and risk of coronary heart disease: the Zutphen Elderly Study. Lancet, 342: 1007-1011, 1993

( 2 ) Knekt P, Jarvinen R, Reunanen A, and Maatela J: Flavonoid intake and coronary mortality in Finland: a cohort study. BMJ, 312: 478-481, 1996

( 3 ) Yochum L, Kushi LH, Meyer K, and Folsom AR: Dietary flavonoid intake and risk of cardiovascular disease in postmenopausal women. Am J Epidemiol, 149: 943-949, 1999

( 4 ) Porter LJ, Ma Z, and Chan BG: Cacao procyanidins; major flavonoids and identification of some minor metabolites. Phytochemistry, 30: 1657-1663, 1991

( 5 ) Adamson GE, Lazarus SA, Mitchell AE, Prior RL, Cao G, Jacobs PH, Kremers BG, Hammerstone JF, Rucker RB, Ritter KA, and Schmitz HH: HPLC method for the quantification of procyanidins in cocoa and chocolate samples and correlation to total antioxidant capacity. J Agric Food Chem, 47: 41844188, 1999

(6) Hatano T, Miyatake H, Natsume M, Osakabe N, Takizawa $\mathrm{T}$, Ito $\mathrm{H}$, and Yoshida $\mathrm{T}$ : Proanthocyanidin glycosides and related polyphenols from cacao liquor and their antioxidant effects. Photochemistry, 59: 749-758, 2002

( 7 ) Osakabe N, Yasuda A, Natsume M, Takizawa T, Terao J, and Kondo K: Catechins and their oligomers linked by $\mathrm{C} 4 \rightarrow \mathrm{C} 8$ bonds are major cacao polyphenols and protect low-density lipoprotein from oxidation in vitro. Exp Biol Med, 227: 51-56, 2002

( 8 ) Lotito SB, Actis-Goretta L, Renart ML, Caligiuri M, Rein D, Schmitz HH, Steinberg FM, Keen CL, and Fraga CG: Influence of oligomer chain length on the antioxidant activity of procyanidins. Biochem Biophys Res Commun, 276: 945-951, 2000 
( 9 ) Osakabe N, Natsume M, Adachi T, Yamagishi M, Hirano R, Takizawa $\mathrm{T}$, Itakura $\mathrm{H}$, and Kondo K: Effects of cacao liquor polyphenols on the susceptibility of low-density lipoprotein to oxidation in hypercholesterolemic rabbits. J Atheroscler Thromb, 7: 164-168, 2000

(10) Osakabe N, Baba S, Yasuda A, Iwamoto T, Kamiyama M, Takizawa $\mathrm{T}$, Itakura $\mathrm{H}$, and Kondo $\mathrm{K}$ : Daily cocoa intake reduces the susceptibility of lowdensity lipoprotein to oxidation as demonstrated in healthy human volunteers. Free Radic Res, 34: 9399, 2001

(11) Steinberg D, Parthasarathy S, Carew TE, Khoo JC, and Witztum JL: Beyond cholesterol. Modifications of low-density lipoprotein that increase its atherogenicity. N Engl J Med, 320: 915-924, 1989

(12) Kurosawa T, Kusanagi M, Yamasaki Y, Senga $Y$, and Yamamoto T: New mutant rabbit strain with hypercholesterolemia and atherosclerotic lesions produced by serial inbreeding. Lab Anim Sci, 45: 385-392, 1995

(13) Yagi K: A simple fluorometric assay for lipoperoxide in blood plasma. Biochem Med, 15: 212-216, 1976

(14) Esterbeaur H, Striegl G, Puhl H, and Rotheneder $\mathrm{M}$ : Continuous monitoring of in vitro oxidation of human low density lipoprotein. Free Radic Res Commun, 6: 67-75, 1989

(15) Hirano R, Kondo K, Iwamoto T, Igarashi O, and Itakura $\mathrm{H}$ : Effects of antioxidants on oxidative susceptibility of low-density lipoprotein. J Nutr Sci Vitaminol (Tokyo), 43: 435-444, 1997

(16) Katsuda S, Machida N, Hasegawa M, Miyashita H, Kusanagi $M$, Tsubone $H$, and Hazama $A$ : Change in the static rheological properties of the aorta in Kurosawa and Kusanagi-Hypercholesterolemic (KHC) rabbits with progress of atherosclerosis. Physiol Meas, 25: 505-522, 2004

(17) da Silva JMR, Darmon N, Fernandez Y, and Mitjavila $\mathrm{S}$ : Oxygen free radical scavenger capacity in aqueous models of different procyanidins from grape seeds. J Agric Food Chem, 39: 1549-1552, 1991

(18) Arteel GE, Schroeder P, and Sies H: Reactions of peroxynitrite with cocoa procyanidin oligomers. J Nutr, 130: 2100S-2104S, 2000

(19) Arteel GE and Sies H: Protection against peroxynitrite by cocoa polyphenol oligomers. FEBS Lett, 462: 167-170, 1999

(20) Baba S, Osakabe N, Natsume M, Yasuda A, Takizawa T, Nakamura T, and Terao J: Cocoa powder enhances the level of antioxidative activity in rat plasma. Br J Nutr, 84: 673-680, 2000

(21) Baba S, Osakabe N, Natsume M, Muto Y, Takizawa $\mathrm{T}$, and Terao J: Absorption and urinary excretion of $(-)$-epicatechin after administration of different levels of cocoa powder or (-)-epicatechin in rats. $J$
Agric Food Chem 49: 6050-6056, 2001

(22) Donovan JL, Crespy V, Manach C, Morand C, Besson C, Scalbert A, and Remesy C: Catechin is metabolized by both the small intestine and liver of rats. J Nutr, 131: 1753-1757, 2001

(23) Baba S, Osakabe N, Yasuda A, Natsume M, Takizawa T, Nakamura T, and Terao J: Bioavailability of (-)-epicatechin upon intake of chocolate and cocoa in human volunteers. Free Radic Res, 33: 635641,2000

(24) Richelle M, Tavazzi I, Enslen M, and Offord EA: Plasma kinetics in man of epicatechin from black chocolate. Eur J Clin Nutr, 53: 22-26, 1999

(25) Newman DL, Gosling RG, and Bowden NLR: Changes in aortic distensibility and area ratio with the development of atherosclerosis. Atherosclerosis, 14: 231-240, 1971

(26) Miyazaki A, Fukuda K, Honda M, Nishimoto Y, Takahashi M, Yamazaki S, Shukuya M, Masuda Y, and Inagaki Y: The study of the physical properties of the aortic wall in the white rabbit fed a high cholesterol diet. Proc Jap Soc Biorhol, 6: 251-254, 1983

(27) Witztum JL: The oxidation hypothesis of atherosclerosis. Lancet, 344: 793-795, 1994

(28) Regnstrom J, Nilsson J, Tornvall P, Landou C, and Hamsten A: Susceptibility to low density lipoprotein oxidation and coronary atherosclerosis in man. Lancet, 339: 1183-1186, 1992

(29) Salonen JT, Yla-Herttuala S, Yamamoto R, Butler S, Korpela H, Salonen R, Nyyssonen K, Palinski W, and Witztum JL: Autoantibody against oxidised LDL and progression of carotid atherosclerosis. Lancet, 339: 883-887, 1992

(30) Kleinveld HA, Demacker PN, and Stalenhoef AF: Comparative study on the effect of low-dose vitamin E and probucol on the susceptibility of LDL to oxidation and the progression of atherosclerosis in Watanabe heritable hyperlipidemic rabbits. Arterioscler Thromb, 14: 1386-1391, 1994

(31) Murakami S, Kondo $\mathrm{Y}$, Sakurai $\mathrm{T}$, Kitajima $\mathrm{H}$, and Nagate T: Taurine suppresses development of atherosclerosis in Watanabe heritable hyperlipidemic (WHHL) rabbit. Atherosclerosis, 163: 79-87, 2002

(32) lizuka A, lijima OT, Yoshie F, Makino B, Amagaya S, Komatsu Y, Kondo K, Matsumoto A, and Itakura $\mathrm{H}$ : Inhibitory effects of Dai-saiko-to (Da-Chai-HuTang) on the progression of atherosclerotic lesions in Kurosawa and Kusanagi-hypercholesterolemic rabbits. J Ethnopharmacol, 63: 209-218, 1998

(33) lizuka A, lijima OT, Kondo K, Matsumoto A, Itakura $\mathrm{H}$, Yoshie F, Komatsu Y, Takeda $\mathrm{H}$, and Matsumiya T: Antioxidative effects of Choi-joki-to and its ability to inhibit the progression of atheroma in $\mathrm{KHC}$ rabbits. J Atheroscler Thromb, 6: 49-54, 2000

(34) Kita T, Nagano Y, Yokode M, Ishii K, Kume N, 
Ooshima A, Yoshida H, and Kawai C: Probucol prevents the progression of atherosclerosis in Watanabe heritable hyperlipidemic rabbit, an animal model for familial hypercholesterolemia. Proc Natl Acad Sci USA, 84: 5928-5931, 1987

(35) Nagano Y, Nakamura T, Matsuzawa Y, Cho M, Ueda $\mathrm{Y}$, and Kita T: Probucol and atherosclerosis in the Watanabe heritable hyperlipidemic rabbit - longterm antiatherogenic effect and effects on established plaques. Atherosclerosis, 92: 131-140, 1992

(36) Yamakoshi J, Kataoka S, Koga T, and Ariga T: Proanthocyanidin-rich extract from grape seeds attenuates the development of aortic atherosclerosis in cholesterol-fed rabbits. Atherosclerosis, 142: 139-149, 1999

(37) Rein D, Paglieroni TG, Wun T, Pearson DA, Schmitz $\mathrm{HH}$, Gosselin R, and Keen CL: Cocoa inhibits platelet activation and function. Am J Clin Nutr, 72: 3035,2000

(38) Karim M, McCormick K, and Kappagoda CT: Effects of cocoa extracts on endothelium-dependent relaxation. J Nutr, 130: 2105S-2108S, 2000

(39) Schramm DD, Wang JF, Holt RR, Ensunsa JL, Gonsalves JL, Lazarus SA, Schmitz HH, German $\mathrm{JB}$, and Keen CL: Chocolate procyanidins decrease the leukotriene-prostacyclin ratio in humans and human aortic endothelial cells. Am J Clin Nutr, 73: 36-40, 2001

(40) Mao TK, Van De Water J, Keen CL, Schmitz HH, and Gershwin ME: Cocoa flavonols and procyanidins promote transforming growth factor$\beta_{1}$ homeostasis in peripheral blood mononuclear cells. Exp Biol Med, 228: 93-99, 2003

(41) Aremu CY, Agiang MA, and Ayatse JO: Nutrient and antinutrient profiles of raw and fermented cocoa beans. Plant Foods Hum Nutr, 48: 217-223, 1995

(42) Jenkins DJA, Kendall CWC, Vuksan V, Vidgen E, Wong E, Augustin LSA, and Fulgoni V: Effect of cocoa bran on low-density lipoprotein oxidation and fecal bulking. Arch Intern Med, 160: 2374-2379, 2000

(43) Das UN: Nutritional factors in the pathobiology of human essential hypertension. Nutrition, 17: 337346, 2001

(44) Massey LK: Dairy food consumption, blood pressure and stroke. J Nutr, 131: 1875-1878, 2001

(45) Cohen H, Sherer Y, Shaish A, Shoenfeld Y, Levkovitz $\mathrm{H}$, Bitzur R, and Harats D: Atherogenesis inhibition induced by magnesium-chloride fortification of drinking water. Biol Trace Elem Res, 90: 251-259, 2002

(46) Sherer Y, Bitzur R, Cohen H, Shaish A, Varon D, Shoenfeld $Y$, and Harats D: Mechanisms of action of the anti-atherogenic effect of magnesium: lessons from a mouse model. Magnes Res, 14: 173179, 2001

(47) Azam S, Hadi N, Khan NU, and Hadi SM: Antioxidant and prooxidant properties of caffeine, theobromine and xanthine. Med Sci Monit, 9: BR325330, 2003 